H. Tanaka

Nagoya Math. J.

Vol. 61 (1976), 85-101

\title{
BOUNDARY VALUE PROBLEMS OF BIHARMONIC FUNCTIONS
}

\author{
HIDEMATU TANAKA
}

\section{Introduction}

Let $\Omega$ be a bounded domain of $n$-dimensional Euclidean space $\boldsymbol{R}^{n}$ $(n \geq 2)$. On $\Omega$ we consider the biharmonic equation

$$
\Delta^{2} u=\left(\sum_{i=1}^{n} \frac{\partial^{2}}{\partial x_{i}^{2}}\right)^{2} u=0 .
$$

A function $u$ in $C^{4}(\Omega)$ is called biharmonic in $\Omega$ if it satisfies the equation (1). In this note we shall deal with the following boundary value problems. Find a biharmonic function $u$ in $\Omega$ such that the following couples of functions have boundary values given on the boundary of $\Omega$ :
(a) $\frac{\partial u}{\partial n}, \frac{\partial(\Delta u)}{\partial n}$;
(b) $\Delta u, \quad \frac{\partial u}{\partial n}$;
(c) $u, \quad \frac{\partial(\Delta u)}{\partial n}$.

J. L. Lions [4] treated these problems for the operator $\Delta^{2}+I$ and gave solutions in case that $\Omega$ is a Nikodym domain. But in his method, the boundary of $\Omega$ or boundary functions are not referred to.

In this note we take as the boundary the Martin boundary $M$ of $\Omega$ and define notations $\gamma_{0}(u)$ and $\gamma_{1}(u)$ for a function $u$ on $\Omega$ as follows. If $u$ has a fine boundary function $f$ on $M$ we denote $f$ by $\gamma_{0}(u)$ and if $u$ has $\varphi$, as generalized normal derivative of Doob [3] (in a slightly modified sense), we denote $\varphi$ by $\gamma_{1}(u)$ (c.f. Definitions 1 and 2).

Now our boundary value problems are described as follows. Find a biharmonic function $u$ in $\Omega$ such that the following couples of functions are equal to boundary functions given on the Martin boundary $M$ :

Received September 7, 1974. 

(a) $\gamma_{1}(u), \quad \gamma_{1}(\Delta u)$;
(b) $\gamma_{0}(\Delta u), \quad \gamma_{1}(u)$;
(c) $\gamma_{0}(u), \quad \gamma_{1}(\Delta u)$.

Let $K(x, \xi)$ be the Martin kernel and $\mu$ be the harmonic measure on $M$. Define new measures $\tilde{\mu}$ and $\tilde{\mu}$ on $M$ by $d \tilde{\mu}(\xi)=k(\xi) d \mu(\xi)$ and $d \tilde{\mu}(\xi)=\frac{1}{k(\xi)} d \mu(\xi)$, where $k(\xi)=\int K(x, \xi) d x$.

Then we shall show that for any $\varphi \in L^{2}(\tilde{\tilde{\mu}})$ with $\int \varphi(\xi) d \mu(\xi)=0$, there exists a square integrable harmonic function $h$ on $\Omega$ with $D(h)<\infty$ such that $\gamma_{1}(h)=\varphi$ if and only if $\Omega$ is a Nikodym domain (Lemma 8). As an application of this fact we shall solve the above boundary value problems as follows.

Assume that $\Omega$ is a Nikodym domain, then

(a) for any $\varphi$ and $\psi$ in $L^{2}(\tilde{\tilde{\mu}})$ with $\int \psi(\xi) d \mu(\xi)=0$ there exists a biharmonic function $u$ such that $\gamma_{1}(u)=\varphi$ and $\gamma_{1}(\Delta u)=\psi$;

(b) for any $f \in L^{2}(\tilde{\mu})$ and $\varphi \in L^{2}(\tilde{\mu})$ with $\int \varphi(\xi) d \mu(\xi)=-\int H_{f}(x) d x$ there exists a biharmonic function $u$ such that $\gamma_{0}(\Delta u)=f$ and $\gamma_{1}(u)=\varphi$;

(c) for any $f \in L^{1}(\mu)$ and $\varphi \in L^{2}(\tilde{\mu})$ with $\int \varphi(\xi) d \mu(\xi)=0$ there exists a biharmonic function $u$ such that $\gamma_{0}(u)=f$ and $\gamma_{1}(\Delta u)=\varphi$.

Moreover the uniqueness of the above solutions will be shown.

\section{Preliminaries}

Let $\Omega$ be an arbitrary bounded domain of the $n$-dimensional Euclidean space $\boldsymbol{R}^{n}(n \geq 2)$ and $G(x, y)$ be it's Green function with respect to the equation $\Delta u=0$, that is $\left(-\Delta_{y}\right) G(x, y)=\varepsilon_{x}$ in $\Omega$.

We shall mention the definition of the Martin boundary of $\Omega$.

We put

$$
K(x, y)=\frac{G(x, y)}{G\left(x_{0}, y\right)}
$$

on $\Omega \times \Omega$ if $y \neq x_{0}$ and $K\left(x, x_{0}\right)=0$ if $x \neq x_{0}$ and $K\left(x_{0}, x_{0}\right)=1$, where $x_{0}$ is a fixed reference point in $\Omega$.

We take a fixed exhaustion $\left\{\Omega_{n}\right\}$ of $\Omega$ such that $x_{0} \in \Omega_{1}$, and put 


$$
d\left(x_{1}, x_{2}\right)=\sum_{n=1}^{\infty} \frac{1}{2^{n}} \sup _{x \in \Omega_{n}}\left|\frac{K\left(x, x_{1}\right)}{1+K\left(x, x_{1}\right)}-\frac{K\left(x, x_{2}\right)}{1+K\left(x, x_{2}\right)}\right| .
$$

Then $d$ defines a metric on $\Omega$. We denote by $\Omega^{*}$ the completion of $\Omega$ by this metric. For a point $\xi \in \Omega^{*}-\Omega$, we can find a sequence $\left\{y_{n}\right\}$ in $\Omega$ such that $d\left(\xi, y_{n}\right) \rightarrow 0$ and so we can define

$$
K(x, \xi)=\lim _{n \rightarrow \infty} K\left(x, y_{n}\right)
$$

We say that $\Omega^{*}$ is the Martin compactification of $\Omega$ and the set $M$ $=\Omega^{*}-\Omega$ is called the Martin boundary of $\Omega$. The function $K(x, \xi)$ on $\Omega \times \Omega^{*}$ is called the Martin kernel. We denote by $\mu$ the harmonic measure on $M$ with respect to the fixed reference point $x_{0}$.

Now let $G_{1}(x, y)$ be the Green function of $\Omega$ with respect to the equation $(\Delta-1) u=0$, that is $\left(-\Delta_{y}+1\right) G_{1}(x, y)=\varepsilon_{x}$ in $\Omega$. For $x \in \Omega$ and $\xi \in M$, we put

$$
K_{1}(x, \xi)=K(x, \xi)-\int G_{1}(x, y) K(y, \xi) d y .
$$

We set for $f \in L^{1}(\mu)$,

$$
H_{f}(x)=\int K(x, \xi) f(\xi) d \mu(\xi)
$$

and

$$
H_{f}^{1}(x)=\int K_{1}(x, \xi) f(\xi) d \mu(\xi) .
$$

Denote by $D(u)$ the Dirichlet integral of $u$ on $\Omega$. For measurable functions $f$ and $g$ on $M$, we put

$$
D(f, g)=\frac{1}{2} \int_{M} \int_{M}(f(\xi)-f(\eta))(g(\xi)-g(\eta)) \theta(\xi, \eta) d \mu(\xi) d \mu(\eta)
$$

and $\boldsymbol{D}(f)=\boldsymbol{D}(f, f)$, where $\theta(\xi, \eta)$ is the Naim kernel (c.f. [7]).

The following lemma is obtained by Doob [3].

LEMMA 1. If $u$ is a harmonic function with $D(u)<\infty$, then $u$ has a fine boundary function $u^{\prime}$ and $\boldsymbol{D}\left(u^{\prime}\right)=D(u)$. Conversely if $f$ is an arbitrary measurable function on $M$ with $D(f)<\infty$, then $f \in L^{2}(\mu)$ and $D\left(H_{f}\right)=D(f)$. 
Put $k(\xi)=\int K(x, \xi) d x$, and $k(\xi)$ is a strictly positive lower semicontinuous function on $M$ and so $\inf _{\xi \in M} k(\xi)=c>0$. Since

$$
\int k(\xi) d \mu(\xi)=\int\left(\int K(x, \xi) d \mu(\xi)\right) d x=|\Omega| \quad(\text { area of } \Omega),
$$

we see that $k(\xi) \in L^{1}(\mu)$.

Define new measures $\tilde{\mu}$ and $\tilde{\mu}$ on $M$ by $d \tilde{\mu}(\xi)=k(\xi) d \mu(\xi)$ and $d \tilde{\mu}(\xi)$ $=\frac{1}{k(\xi)} d \mu(\xi)$ respectively, and we have the following relations

$$
B(M) \subset L^{2}(\tilde{\mu}) \subset L^{2}(\mu) \subset L^{2}(\tilde{\mu}) \subset L^{1}(\mu),
$$

where $\boldsymbol{B}(M)$ is the space of all bounded measurable functions on $M$. We also see that

$$
\|f\|_{L^{2}(\tilde{\tilde{\mu}})} \leq \frac{1}{\sqrt{c}}\|f\|_{L^{2}(\mu)} \leq \frac{1}{c}\|f\|_{L^{2}(\tilde{\mu})}
$$

for any $f \in L^{2}(\tilde{\mu})$.

By the Fubini theorem, $\int H_{f^{2}}(x) d x<\infty$ for any $f \in L^{2}(\tilde{\mu})$. Hence we know

$$
\begin{aligned}
\int H_{|f|}(x) H_{|g|}^{1}(x) d x & \leq \int H_{|f|}(x) H_{|g|}(x) d x \\
& \leq\left(\int\left(H_{|f|}(x)\right)^{2} d x \cdot \int\left(H_{|g|}(x)\right)^{2} d x\right)^{1 / 2} \\
& \leq\left(\int H_{f^{2}}(x) d x \cdot \int H_{g^{2}}(x) d x\right)^{1 / 2}<\infty
\end{aligned}
$$

for any $f$ and $g$ in $L^{2}(\tilde{\mu})$.

LEMMA 2. Let $f$ and $g$ be in $L^{2}(\tilde{\mu})$. Then

$$
\int H_{f}(x) H_{g}^{1}(x) d x=\int H_{g}(x) H_{f}^{1}(x) d x
$$

and

$$
\int H_{f}(x) H_{f}^{1}(x) d x \leq \int\left(H_{f}(x)\right)^{2} d x \leq c^{\prime} \cdot \int H_{f}(x) H_{f}^{1}(x) d x
$$

for some constant $c^{\prime} \geq 1$.

Proof. By the definition of $K_{1}(x, \xi)$ and the resolvent equation, 


$$
H_{f}^{1}(x)=H_{f}(x)-\int G_{1}(x, y) H_{f}(y) d y
$$

and

$$
H_{f}(x)=H_{f}^{1}(x)+\int G(x, y) H_{f}^{1}(y) d y
$$

Hence

$$
\begin{aligned}
\int H_{g}(x) H_{f}^{1}(x) d x & =\int H_{g}(x)\left(H_{f}(x)-\int G_{1}(x, y) H_{f}(y) d y\right) d x \\
& =\int H_{g}(x) H_{f}(x) d x-\int H_{f}(y)\left(\int G_{1}(x, y) H_{g}(x) d x\right) d y \\
& =\int H_{g}(x) H_{f}(x) d x-\int H_{f}(y)\left(H_{g}(y)-H_{g}^{1}(y)\right) d y \\
& =\int H_{f}(x) H_{g}^{1}(x) d x
\end{aligned}
$$

and

$$
\begin{aligned}
\int\left(H_{f}(x)\right)^{2} d x-\int H_{f}(x) H_{f}^{1}(x) d x & =\int H_{f}(x)\left(H_{f}(x)-H_{f}^{1}(x)\right) d x \\
& =\int H_{f}(x)\left(\int G_{1}(x, y) H_{f}(y) d y\right) d x \\
& =\iint G_{1}(x, y) H_{f}(x) H_{f}(y) d x d y \geq 0 .
\end{aligned}
$$

By (11)

$$
\int\left(H_{f}(x)\right)^{2} d x-\int H_{f}(x) H_{f}^{1}(x) d x=\int H_{f}(x)\left(\int G(x, y) H_{f}^{1}(y) d y\right) d x
$$

and hence

$$
\begin{aligned}
& \left(\int\left(H_{f}(x)\right)^{2} d x-\int H_{f}(x) H_{f}^{1}(x) d x\right)^{2} \\
& \quad \leq \int\left(H_{f}(x)\right)^{2} d x \cdot\left(\int\left(\int G(x, y) d y \cdot \int G(x, y)\left(H_{f}^{1}(y)\right)^{2} d y\right) d x\right) \\
& \quad \leq c_{0}^{2} \cdot \int\left(H_{f}(x)\right)^{2} d x \cdot \int\left(H_{f}^{1}(x)\right)^{2} d x
\end{aligned}
$$

where $c_{0}=\sup _{x \in \Omega} \int G(x, y) d y$. Similarly to (12), we know

$$
\int H_{f}(x) H_{f}^{1}(x) d x-\int\left(H_{f}^{1}(x)\right)^{2} d x \geq 0,
$$


and so we have an inequality

$$
\begin{aligned}
& \int\left(H_{f}(x)\right)^{2} d x-\int H_{f}(x) H_{f}^{1}(x) d x \\
& \quad \leq c_{0} \cdot\left(\int\left(H_{f}(x)\right)^{2} d x\right)^{1 / 2}\left(\int H_{f}(x) H_{f}^{1}(x) d x\right)^{1 / 2} .
\end{aligned}
$$

Hence

$$
\int\left(H_{f}(x)\right)^{2} d x \leq c^{\prime} \cdot \int H_{f}(x) H_{f}^{1}(x) d x
$$

for some constant $c^{\prime} \geq 1$. This completes the proof.

Now we set

$$
\tilde{\boldsymbol{H}}(M)=\left\{f ; f \in \boldsymbol{L}^{2}(\tilde{\mu}) \text { and } \boldsymbol{D}(f)<\infty\right\},
$$

and define two inner products on $\tilde{\boldsymbol{H}}(M)$ by

$$
(f, g)_{1}=\boldsymbol{D}(f, g)+\int H_{f}(x) H_{g}(x) d x
$$

and

$$
(f, g)_{2}=D(f, g)+\int H_{f}(x) H_{g}^{1}(x) d x
$$

for functions $f$ and $g$ in $\tilde{\boldsymbol{H}}(M)$. By the above lemma, we know that $(\cdot, \cdot)_{2}$ is an inner product on $\tilde{\boldsymbol{H}}(M)$. We put $\|f\|_{1}^{2}=(f, f)_{1}$ and $\|f\|_{2}^{2}=$ $(f, f)_{2}$ for $f \in \tilde{H}(M)$. Then we have

LEMMA 3. Norms $\|\cdot\|_{1}$ and $\|\cdot\|_{2}$ are equivalent and $\tilde{\boldsymbol{H}}(M)$ is a Hilbert space with respect to these norms.

Proof. By the above lemma,

$$
\|f\|_{2} \leq\|f\|_{1} \leq\left(\max \left(1, c^{\prime}\right)\right)^{1 / 2}\|f\|_{2},
$$

and so these norms are equivalent. Let $f$ be in $\tilde{\boldsymbol{H}}(M)$. Then by the Riesz decomposition of $-\left(H_{f}\right)^{2}$ we have

$$
\left(H_{f}\right)^{2}=H_{f^{2}}-\int G(\cdot, y) d \nu_{f}(y) \text {. }
$$

Since $D\left(H_{f}\right)=\frac{1}{2} \int d \nu_{f}$, we have 


$$
\begin{aligned}
\|f\|_{L^{2}(\tilde{\mu})}^{2} & =\int H_{f 2}(x) d x \\
& =\int\left(\left(H_{f}(x)\right)^{2}+\int G(x, y) d \nu_{f}(y)\right) d x \\
& \leq \int\left(H_{f}(x)\right)^{2} d x+c_{0} \cdot \int d \nu_{f} \\
& \leq \max \left(1,2 c_{0}\right)\left(\int\left(H_{f}(x)\right)^{2} d x+D\left(H_{f}\right)\right) \\
& =\max \left(1,2 c_{0}\right)\left(\int\left(H_{f}(x)\right)^{2} d x+D(f)\right) \\
& =\max \left(1,2 c_{0}\right)\|f\|_{1}^{2} .
\end{aligned}
$$

Hence we see that $\tilde{\boldsymbol{H}}(M)$ is a Hilbert space.

3. Definitions of $\gamma_{0}(u)$ and $\gamma_{1}(u)$ for a function $u$ on $\Omega$

We shall define $\gamma_{0}(u)$ and $\gamma_{1}(u)$ for a function $u$ on $\Omega$ as follows.

DEFINITION 1. If a function $u$ on $\Omega$ has a fine boundary function $f$ on $M$, we denote $f$ by $\gamma_{0}(u)$.

The definition of $\gamma_{1}(u)$ is a slight modification of the definition of the generalized normal derivative of $u$ (c.f. Doob [3]).

DEFinition 2. Consider the function $u(x)=H_{f}(x)+u_{p}(x)$, where $f$ is a measurable function on $M$ with $D(f)<\infty$ and $u_{p}$ is a potential of a measure $\nu$ on $\Omega$. We assume that for any $g \in \boldsymbol{H}(M), H_{g}$ is integrable on $\Omega$ with respect to the absolute variation of $\nu$. If there exists a function $\varphi$ on $M$ such that $\int \varphi(\xi) g(\xi) d \mu(\xi)<+\infty$ and

$$
D(f, g)=-\int \varphi(\xi) g(\xi) d \mu(\xi)+\int H_{g}(x) d \nu(x)
$$

for any $g \in \tilde{\boldsymbol{H}}(M)$, we denote $\varphi$ by $\gamma_{1}(u)$.

We shall show the following

LEMMA 4. Let $\varphi$ be in $L^{2}(\tilde{\tilde{\mu}})$. Then there exists a unique function $f \in \tilde{\boldsymbol{H}}(M)$ such that $\gamma_{1}(u)=\varphi$, where

$$
u(x)=H_{f}(x)-\int G(x, y) H_{f}(y) d y .
$$

Proof. In the Hilbert space $\tilde{\boldsymbol{H}}(M)$ with the norm $\|\cdot\|_{1}$, the mapping 
$g \rightarrow-\int g(\xi) \varphi(\xi) d \mu(\xi)$ is a linear functional. By the Schwarz inequality and (17), we have

$$
\begin{aligned}
\left|-\int g(\xi) \varphi(\xi) d \mu(\xi)\right|^{2} & \leq\left(\int|g(\xi)| k(\xi)^{1 / 2} \frac{1}{k(\xi)^{1 / 2}}|\varphi(\xi)| d \mu(\xi)\right)^{2} \\
& \leq\|\varphi\|_{L^{2}(\tilde{\tilde{\mu}})}^{2} \cdot\|g\|_{L^{2}(\tilde{\mu})}^{2} \\
& \leq \max \left(1,2 c_{0}\right)\|\varphi\|_{L^{2}(\tilde{\tilde{\mu}})}^{2} \cdot\|g\|_{1}^{2} .
\end{aligned}
$$

Hence the above mapping is bounded on $\tilde{H}(M)$. Therefore there exists a unique function $f \in \tilde{\boldsymbol{H}}(M)$ such that $(f, g)_{1}=-\int \varphi(\xi) g(\xi) d \mu(\xi)$, namely

$$
D(f, g)=-\int \varphi(\xi) g(\xi) d \mu(\xi)+\int H_{g}(x)\left(-H_{f}(x)\right) d x
$$

for any $g \in \tilde{H}(M)$. If we put $u(x)=H_{f}(x)-\int G(x, y) H_{f}(y) d y$, then from the definition we have $\gamma_{1}(u)=\varphi$.

Similarly we have

LEMMA 5. Let $\varphi$ be in $L^{2}(\tilde{\mu})$. Then there exists a unique function $f \in \tilde{\boldsymbol{H}}(M)$ such that $\gamma_{1}\left(H_{f}^{1}\right)=\varphi$.

Proof. By Lemma 3, the mapping $g \rightarrow-\int g(\xi) \varphi(\xi) d \mu(\xi)$ is a bounded linear functional on the Hilbert space $\tilde{\boldsymbol{H}}(M)$ with the norm $\|\cdot\|_{2}$. Hence there exists a unique function $f \in \tilde{H}(M)$ such that

$$
D(f, g)=-\int \varphi(\xi) g(\xi) d \mu(\xi)+\int H_{g}(x)\left(-H_{f}^{1}(x)\right) d x
$$

for any $g \in \tilde{\boldsymbol{H}}(M)$. Since $H_{f}^{1}(x)=H_{f}(x)-\int G(x, y) H_{f}^{1}(y) d y$, we have $\gamma_{1}\left(H_{f}^{1}\right)$ $=\varphi$.

We set

$$
\widehat{\boldsymbol{H}(M)}=\left\{f \in \tilde{\boldsymbol{H}}(M) ; \text { there exists } \gamma_{1}\left(H_{f}\right) \in L^{2}(\tilde{\mu})\right\}
$$

Then we have similarly to Folgesatz 17.27 in [1] and Theorem 6 in [6] the following

LEMMA 6. $\widehat{\boldsymbol{H}(M)}$ is dense in $\tilde{\boldsymbol{H}}(M)$. 
Proof. Let $f_{0}$ be in $\tilde{\boldsymbol{H}}(M)$ and $\left(f_{0}, g\right)_{1}=0$ for any $g \in \tilde{\boldsymbol{H}}(M)$. Then we have

$$
D\left(f_{0}, g\right)+\int H_{f_{0}}(x) H_{g}(x) d x=0 .
$$

Since $f_{0}$ is in $L^{2}(\tilde{\mu})$, by Lemma 4 there exists $f_{0}^{\prime} \in \tilde{\boldsymbol{H}}(M)$ such that

$$
\gamma_{1}\left(H_{f_{0}^{\prime}}-\int G(\cdot, y) H_{f_{0}^{\prime}}(y) d y\right)=f_{0} .
$$

On the other hand

$$
\gamma_{1}\left(\int G(\cdot, y) H_{f_{0}^{\prime}}(y) d y\right)=\int K(x, \cdot) H_{f_{0}^{\prime}}(x) d x
$$

and

$$
\left\|\int K(x, \cdot) H_{f_{0}^{\prime}}(x) d x\right\|_{L^{2}(\tilde{\tilde{\mu}})} \leq\left\|f_{0}^{\prime}\right\|_{L^{2}(\tilde{\mu})}<\infty .
$$

Hence $\gamma_{1}\left(H_{f_{0}^{\prime}}\right) \in \boldsymbol{L}^{2}(\tilde{\mu})$ and $f_{0}^{\prime}$ is in $\left.\widehat{H(M}\right)$. By (19), we have

$$
\boldsymbol{D}\left(f_{0}, f_{0}^{\prime}\right)+\int H_{f_{0}}(x) H_{f_{0}^{\prime}}(x) d x=0
$$

and by (20),

$$
\boldsymbol{D}\left(f_{0}, f_{0}^{\prime}\right)=-\int f_{0}^{2}(\xi) d \mu(\xi)-\int H_{f_{0}}(x) H_{f_{0}^{\prime}}(x) d x
$$

therefore we know that $f_{0}=0$. This completes the proof.

\section{Nikodym domain}

In this section we shall treat the problem whether we are able to find $f \in \tilde{\boldsymbol{H}}(M)$ such that $\gamma_{1}\left(H_{f}\right)=\varphi$ for any $\varphi \in \boldsymbol{L}^{2}(\tilde{\tilde{\mu}})$ with $\int \varphi(\xi) d \mu(\xi)=0$.

Definition 3. (Deny-Lions [2]) We shall say that $\Omega$ is a Nikodym domain if every distribution $T$ with $\frac{\partial}{\partial x_{i}} T \in L^{2}(\Omega)(1 \leq i \leq n)$ is in $L^{2}(\Omega)$.

We set $\mathscr{E}_{L^{2}}^{I_{1}}(\Omega)=\left\{u ; u \in \boldsymbol{L}^{2}(\Omega)\right.$ and $\left.\frac{\partial}{\partial x_{i}} u \in \boldsymbol{L}^{2}(\Omega)(1 \leq i \leq n)\right\}$

A necessary and sufficient condition for $\Omega$ to be a Nikodym domain is given by the following inequality of Poincaré: there exists a constant $P(\Omega)$ such that 


$$
\int(u(x))^{2} d x-\frac{1}{|\Omega|}\left|\int u(x) d x\right|^{2} \leq P(\Omega) D(u)
$$

for any $u \in \mathscr{E}_{L^{2}}^{1}(\Omega)$ (c.f. [2]).

Deny-Lions [2] gives another characterization of a Nikodym domain by setting

$$
\boldsymbol{N}=\left\{\begin{array}{c}
u \in \mathscr{E}_{L^{2}}^{1}(\Omega) ; \Delta u \in L^{2}(\Omega) \text { and }(-\Delta u, v)_{L^{2}(\Omega)}=D(u, v) \\
\text { for any } v \in \mathscr{E}_{L^{2}}^{1}(\Omega)
\end{array}\right\} .
$$

LEMMA 7. (Deny-Lions) For any $F \in L^{2}(\Omega)$ with $\int F(x) d x=0$ we can find $u$ in $N$ (unique up to an additive constant) such that $-\Delta u=F$ if and only if $\Omega$ is a Nikodym domain.

The following lemma gives an answer to our above problem and it gives a characterization of a Nikodym domain.

LeMma 8. For any $\varphi \in L^{2}(\tilde{\mu})$ with $\int \varphi(\xi) d \mu(\xi)=0$ we can find $f$ in $\tilde{\boldsymbol{H}}(M)$ (unique up to an additive constant) such that $\gamma_{1}\left(H_{f}\right)=\varphi$ if and only if $\Omega$ is a Nikodym domain.

Proof. Assume that $\Omega$ is a Nikodym domain. Let $\varphi$ be in $L^{2}(\tilde{\mu})$ with $\int \varphi(\xi) d \mu(\xi)=0$. Then by Lemma 4 there exists a unique function $f_{0} \in \tilde{\boldsymbol{H}}(M)$ such that

$$
\gamma_{1}\left(H_{f_{0}}-\int G(\cdot, y) H_{f_{0}}(y) d y\right)=\varphi
$$

Hence

$$
D\left(f_{0}, g\right)=-\int \varphi(\xi) g(\xi) d \mu(\xi)+\int H_{g}(x)\left(-H_{f_{0}}(x)\right) d x
$$

for any $g \in \tilde{H}(M)$. We put $g=1$ in (21), then $\int H_{f_{0}}(x) d x=0$ from the condition $\int \varphi(\xi) d \mu(\xi)=0$.

Since $f_{0}$ is in $\tilde{\boldsymbol{H}}(M), H_{f_{0}} \in L^{2}(\Omega)$ and $D\left(H_{f_{0}}\right)=D\left(f_{0}\right)<\infty$. Therefore by Lemma 7 , we can find $u$ in $N$ (unique up to an additive constant) such that $-\Delta u=H_{f_{0}}$. Hence we know that $\Delta^{2} u=0, u \in L^{2}(\Omega)$ and $D(u)$ $<\infty$ and so by the uniqueness of the Royden decomposition of $u$, we have 


$$
\begin{aligned}
u(x) & =h(x)-\int G(x, y) \Delta u(y) d y \\
& =h(x)+\int G(x, y) H_{f_{0}}(y) d y
\end{aligned}
$$

for some harmonic function $h \in \boldsymbol{L}^{2}(\Omega)$ with $D(h)<\infty$. From (17), $h$ has a fine boundary function $h^{\prime}$ in $L^{2}(\tilde{\mu})$ and so $h=H_{h^{\prime}}$ with $h^{\prime} \in \tilde{\boldsymbol{H}}(M)$.

Since $u$ is in $N$ and $\left\{H_{g} ; g \in \tilde{H}(M)\right\} \subset \mathscr{E}_{L^{2}}^{1}(\Omega)$, we have

$$
\int H_{g}(x)(-\Delta u(x)) d x=D\left(u, H_{g}\right)
$$

for any $g \in \tilde{\boldsymbol{H}}(M)$. Hence we have

$$
\begin{aligned}
\boldsymbol{D}\left(h^{\prime}, g\right) & -\int H_{g}(x) H_{f_{0}}(x) d x \\
= & D\left(h, H_{g}\right)-\int H_{g}(x)(-\Delta u(x)) d x \\
= & D\left(h, H_{g}\right)-D\left(u, H_{g}\right) \\
= & D\left(h-u, H_{g}\right) \\
= & D\left(\int G(\cdot, y) \Delta u(y) d y, H_{g}\right)=0
\end{aligned}
$$

for any $g \in \tilde{\boldsymbol{H}}(M)$ and so $\gamma_{1}(u)=0$.

Now we put $f=f_{0}+h^{\prime}$, then $f$ is determined (uniquely up to an additive constant) in $\tilde{\boldsymbol{H}}(M)$ and we have

$$
\begin{aligned}
\gamma_{1}\left(H_{f}\right) & =\gamma_{1}\left(H_{f_{0}}+h\right) \\
& =\gamma_{1}\left(H_{f_{0}}-\int G(\cdot, y) H_{f_{0}}(y) d y+u\right) \\
& =\varphi .
\end{aligned}
$$

Conversely assume that for any $\varphi \in L^{2}(\tilde{\tilde{\mu}})$ with $\int \varphi(\xi) d \mu(\xi)=0$ we can find $f$ in $\tilde{\boldsymbol{H}}(M)$ such that $\gamma_{1}\left(H_{f}\right)=\varphi$. We shall show that for any $v$ $\in L^{2}(\Omega)$ with $\int v(x) d x=0$, we can find $u$ in $N$ (unique up to an additive constant) such that $-\Delta u=v$. Then by Lemma 7 we conclude that $\Omega$ is a Nikodym domain. Let $v$ be in $L^{2}(\Omega)$ with $\int v(x) d x=0$. Since

$$
\int|v(x)| \cdot\left|H_{g}(x)\right| d x<\infty
$$

for any $g \in \tilde{H}(M)$, we know 


$$
\gamma_{1}\left(-\int G(\cdot, y) v(y) d y\right)=-\int K(x, \cdot) v(x) d x
$$

Put $\varphi_{v}=\gamma_{1}\left(-\int G(\cdot, y) v(y) d y\right)$, and we know

$$
\begin{aligned}
& \int \varphi_{v}^{2}(\xi) d \tilde{\tilde{\mu}}(\xi)=\int \frac{1}{k(\xi)} \varphi_{v}^{2}(\xi) d \mu(\xi) \\
& \leq \int \frac{1}{k(\xi)}\left(\int K(x, \xi) d x \cdot \int K(x, \xi) v^{2}(x) d x\right) d \mu(\xi) \\
& \quad=\|v\|_{L^{2}(\Omega)}^{2}<\infty
\end{aligned}
$$

and

$$
\int \varphi_{v}(\xi) d \mu(\xi)=\int\left(-\int K(x, \xi) v(x) d x\right) d \mu(\xi)=-\int v(x) d x=0
$$

Hence we can find $f$ in $\tilde{H}(M)$ (unique up to an additive constant) such that $\gamma_{1}\left(H_{f}\right)=\varphi_{v}$. We put

$$
u(x)=H_{f}(x)+\int G(x, y) v(y) d y
$$

thus $u$ is determined (uniquely up to an additive constant) in $\mathscr{E}_{L^{2}}^{1}(\Omega)$, $-\Delta u=v$ and $\Delta u \in L^{2}(\Omega)$.

Now we shall show that $u$ is in $N$, that is $D(u, w)=(-\Delta u, w)_{L^{2}(\Omega)}$ for any $w$ in $\mathscr{E}_{L^{2}}^{1}(\Omega)$.

We have the following decomposition of $\mathscr{E}_{L^{2}}^{1}(\Omega)$ :

$$
\mathscr{E}_{L^{2}}^{1}(\Omega)=\left\{H_{g}^{1} ; g \in \tilde{\boldsymbol{H}}(M)\right\} \oplus L^{2} D_{0}(\Omega),
$$

where $L^{2} D_{0}(\Omega)$ is the closure of $C_{0}^{\infty}(\Omega)$ with respect to the norm $D(\cdot)$ $+\|\cdot\|_{L^{2}(\Omega)}$. In case $w=H_{g}^{1}$ for some $g \in \tilde{H}(M)$, we have

$$
\begin{aligned}
D(u, w) & =D\left(u, H_{g}^{1}\right) \\
& =D\left(H_{f}, H_{g}\right)-D\left(\int G(\cdot, y) v(y) d y, \int G(\cdot, y) H_{g}^{1}(y) d y\right) \\
& =D(f, g)-\int v(x)\left(\int G(x, y) H_{g}^{1}(y) d y\right) d x .
\end{aligned}
$$

Since $\gamma_{1}(u)=\gamma_{1}\left(H_{f}\right)+\int K(x, \cdot) v(x) d x=\varphi_{v}-\varphi_{v}=0$, we know

$$
D(f, g)=\int v(x) H_{g}(x) d x
$$


for any $g \in \tilde{\boldsymbol{H}}(M)$. Hence we have

$$
\begin{aligned}
D\left(u, H_{g}^{1}\right) & =\int v(x)\left(H_{g}(x)-\int G(x, y) H_{g}^{1}(y) d y\right) d x \\
& =-\int \Delta u(x) H_{g}^{1}(x) d x .
\end{aligned}
$$

In case $w$ is in $C_{0}^{\infty}(\Omega)$ we know that

$$
w(x)=\int G(x, y)(-\Delta w(y)) d y .
$$

Hence

$$
\begin{aligned}
D(u, w) & =D\left(\int G(\cdot, y) v(y) d y, \int G(\cdot, y)(-\Delta w(y)) d y\right) \\
& =\int v(x)\left(\int G(x, y)(-\Delta w(y)) d y\right) d x \\
& =-\int \Delta u(x) w(x) d x .
\end{aligned}
$$

For any $w$ in $L^{2} D_{0}(\Omega)$, we can find a sequence $\left\{w_{n}\right\}$ in $C_{0}^{\infty}(\Omega)$ such that $w_{n} \rightarrow w$ in $L^{2} D_{0}(\Omega)$. Since $D\left(u, w_{n}\right)=-\int \Delta u(x) w_{n}(x) d x$, letting $n \rightarrow \infty$, we have $D(u, w)=-\int \Delta u(x) w(x) d x$. Therefore we know

$$
D(u, w)=(-\Delta u, w)_{L^{2}(\Omega)}
$$

for any $w \in \mathscr{E}_{L^{2}}^{1}(\Omega)$ and so $u$ is in $N$. This completes the proof.

\section{Boundary value problems}

In this section we shall solve the boundary value problems described in section 1 as an application of Lemma 8. We put

$$
\begin{aligned}
& \mathscr{S}_{1}=\left\{u \in C^{4}(\Omega) ; u \text { and } \Delta u \text { are in } \mathscr{E}_{L^{2}}^{1}(\Omega)\right\}, \\
& \mathscr{S}_{2}=\left\{u \in C^{4}(\Omega) ; u \text { is in } \mathscr{E}_{L^{2}}^{1}(\Omega) \text { and } \Delta u \text { is in } L^{2}(\Omega)\right\}
\end{aligned}
$$

and

$$
\mathscr{S}_{3}=\left\{u \in C^{4}(\Omega) ; \Delta u \text { is in } \mathscr{E}_{L^{2}}^{1}(\Omega)\right\} .
$$

Then we shall show

THEOREM. Assume that $\Omega$ is a Nikodym domain, then

(a) for any $\varphi$ and $\psi$ in $L^{2}(\tilde{\tilde{\mu}})$ with $\int \psi(\xi) d \mu(\xi)=0$, there exists $u$ in 
$\mathscr{S}_{1}$ unique up to an additive constant such that $\Delta^{2} u=0, \gamma_{1}(u)=\varphi$ and $\gamma_{1}(\Delta u)=\psi$

(b) for any $f$ in $L^{2}(\tilde{\mu})$ and $\varphi$ in $L^{2}(\tilde{\mu})$ with

$$
\int \varphi(\xi) d \mu(\xi)=-\int H_{f}(x) d x
$$

there exists $u$ in $\mathscr{S}_{2}$ unique up to an additive constant such that $\Delta^{2} u$ $=0, \gamma_{0}(\Delta u)=f$ and $\gamma_{1}(u)=\varphi$;

(c) for any $f$ in $L^{1}(\mu)$ and $\varphi$ in $L^{2}(\tilde{\mu})$ with $\int \varphi(\xi) d \mu(\xi)=0$, there exists $u$ in $\mathscr{S}_{3}$ such that $\Delta^{2} u=0, \gamma_{0}(u)=f$ and $\gamma_{1}(\Delta u)=\varphi$.

Proof. (a) For any $\varphi$ and $\psi$ in $L^{2}(\tilde{\mu})$ with $\int \psi(\xi) d \mu(\xi)=0$, by Lemma 8 there exists $f$ in $\tilde{\boldsymbol{H}}(M)$ such that $\gamma_{1}\left(H_{f}\right)=\psi$ and

$$
\int\left(\varphi(\xi)+\int K(x, \xi) H_{f}(x) d x\right) d \mu(\xi)=0 .
$$

Since $\varphi+\int K(x, \cdot) H_{f}(x) d x$ is in $L^{2}(\tilde{\mu})$ and (23), there exists $f_{0}$ in $\tilde{\boldsymbol{H}}(M)$ such that $\gamma_{1}\left(H_{f_{0}}\right)=\varphi+\int K(x, \cdot) H_{f}(x) d x$.

We put

$$
u(x)=H_{f_{0}}(x)-\int G(x, y) H_{f}(y) d y .
$$

Then we know that $u$ is in $\mathscr{S}_{1}, \Delta^{2} u=0, \gamma_{1}(u)=\varphi$ and $\gamma_{1}(\Delta u)=\psi$.

Next we shall show the uniqueness of the solution. Let $w$ be in $\mathscr{S}_{1}$ such that $\Delta^{2} w=0, \gamma_{1}(w)=0$ and $\gamma_{1}(\Delta w)=0$. By the uniqueness of the Royden decomposition of $w$, there exists $f_{w}$ and $g_{w}$ in $\tilde{\boldsymbol{H}}(M)$ such that

$$
w=H_{f_{w}}-\int G(\cdot, y) \Delta w(y) d y
$$

and $\Delta w=H_{g_{w}}$. Since $\gamma_{1}(w)=0$, we have

$$
D\left(H_{f_{w}}, H_{\vartheta}\right)+\int \Delta w(x) H_{g}(x) d x=0
$$

for any $g$ in $\tilde{\boldsymbol{H}}(M)$. Hence 


$$
\begin{aligned}
D(w, w) & =D\left(H_{f_{w}}, H_{f_{w}}\right)+\iint G(x, y) \Delta w(x) \Delta w(x) d x d y \\
& =-\int \Delta w(x) H_{f_{w}}(x) d x+\int \Delta w(x)\left(\int G(x, y) \Delta w(y) d y\right) d x \\
& =-\int \Delta w(x) w(x) d x .
\end{aligned}
$$

Since $\gamma_{1}(\Delta w)=0$, we have

$$
D\left(\Delta w, H_{g}\right)=0
$$

for any $g$ in $\tilde{\boldsymbol{H}}(M)$. We put $g=g_{w}$ in (24) and $g=f_{w}$ in (26), then we know that $\Delta w=0$ and so $w=$ constant by (25).

(b) First we shall remark that the condition (22) is necessary for the existence of the solution. Let $u$ be a solution, then

$$
u(x)=H_{f_{u}}(x)-\int G(x, y) \Delta u(y) d y
$$

for some $f_{u} \in \tilde{\boldsymbol{H}}(M)$. Since $\gamma_{0}(\Delta u)=f$ and $\gamma_{1}(u)=\varphi$, we know $\Delta u=H_{f}$ and

$$
D\left(H_{f_{u}}, H_{g}\right)=-\int \varphi(\xi) g(\xi) d \mu(\xi)+\int H_{g}(x)(-\Delta u(x)) d x
$$

for any $g \in \tilde{H}(M)$. Put $g=1$ in (27) and we have (22).

For any $f$ in $L^{2}(\tilde{\mu})$ and $\varphi$ in $L^{2}(\tilde{\tilde{\mu}})$ we know that $\int K(x, \cdot) H_{f}(x) d x$ is in $L^{2}(\tilde{\mu})$ and by $(22)$

$$
\int\left(\varphi(\xi)+\int K(x, \xi) H_{f}(x) d x\right) d \mu(\xi)=0 .
$$

Hence there exists $f_{0}$ in $\tilde{\boldsymbol{H}}(M)$ such that

$$
\gamma_{1}\left(H_{f_{0}}\right)=\varphi+\int K(x, \cdot) H_{f}(x) d x
$$

We put

$$
u(x)=H_{f_{0}}(x)-\int G(x, y) H_{f}(y) d y
$$

Then $u$ is in $\mathscr{S}_{2}, \Delta^{2} u=0, \gamma_{0}(\Delta u)=f$ and $\gamma_{1}(u)=\varphi$.

The uniqueness of the solution is shown in a similar manner to (a). Let $w$ be in $\mathscr{S}_{2}$ such that $\Delta^{2} w=0, \gamma_{0}(\Delta w)=0$ and $\gamma_{1}(w)=0$, then we have 


$$
D(w, w)+\int \Delta w(x) w(x) d x=0
$$

Since $\Delta w$ is harmonic and $\gamma_{0}(\Delta w)=0$, we know $\Delta w=0$ and so $w=$ constant.

(c) Put

$$
u(x)=H_{f}(x)-\int G(x, y) H_{f_{0}}(y) d y
$$

where $f_{0}$ is in $\tilde{\boldsymbol{H}}(M)$ such that $\gamma_{1}\left(H_{f_{0}}\right)=\varphi$, and $u$ is the desired solution. This completes the proof.

Remark 1. In the case of (c) the uniqueness of the solution is interpreted as follows. If $u_{0}$ is a solution of $(c)$, then every solution is given by $u_{0}+a \int G(\cdot, y) d y$, where $a$ is some constant.

In fact if $w$ is in $\mathscr{S}_{3}, \Delta^{2} w=0, \gamma_{0}(w)=0$ and $\gamma_{1}(\Delta w)=0$, then $h(x)$ $=w(x)+\int G(x, y) \Delta w(y) d y$ is harmonic and $\gamma_{0}(h)=0$. Hence we have $w(x)=-\int G(x, y) \Delta w(y) d y$. Since $\gamma_{1}(\Delta w)=0$, we know $w(x)=a \int G(x, y) d y$ for some constant $a$.

Remark 2. Lemma 8 asserts that if one of the above boundary value problems has always a solution, then $\Omega$ is necessarily a Nikodym domain. Hence the above problems are solved if and only if $\Omega$ is a Nikodym domain.

\section{REFERENCES}

[1] C. Constantinescu and A. Cornea, Ideale Ränder Riemannscher Flächen. Spriger Verlag (1963).

[2] J. Deny and J. L. Lions, Les espaces du type de Beppo Levi. Ann. Inst. Fourier, 5 (1953-4), 305-370.

[ 3 ] J. L. Doob, Boundary properties of functions with finite Dirichlet integrals. Ann. Inst. Fourier, 12 (1962), 573-621.

[4] J. L. Lions, Sur quelques problèmes aux limites relatifs a des opérateurs différentiels elliptiques. Bull. Soc. Math. France, 83 (1955), 225-250.

[ 5 ] — Problèmes aux limites en théorie des distributions. Acta Math. 94 (1955), 13-153.

[6] F.-Y. Maeda, Normal derivatives on an ideal boundary. J. Sci. Hiroshima Univ. Ser. A-1, 28 (1964), 113-131.

[ 7 ] L. Naïm, Sur le rôle de la frontière de R. S. Martin dans la théorie du potentiel. Ann. Inst. Fourier, 7 (1957), 183-281. 
[8] M. Nakai, Dirichlet finite biharmonic functions with Dirichlet finite laplacians. Math. Z., 122 (1971), 203-216.

Department of Mathematics

Saitama University 